\title{
Proposed SecureSIP Authentication Scheme based on Elliptic Curve Cryptography
}

\author{
Samaneh Sadat \\ Mousavi-Nik \\ MSc. of IT - Information Security \\ Tehran University \\ Tehran, Iran
}

\author{
M.H. Yaghmaee- \\ Moghaddam \\ School of Engineering \\ Ferdowsi University
}

\author{
M.B. Ghaznavi- \\ Ghoushchi \\ School of Engineering \\ Shahed University \\ Tehran, Iran
}

\begin{abstract}
Session Initiation Protocol (SIP) is a powerful signaling protocol that increasingly used for administrating Voice over IP (VoIP) phone calls. In recent years, Session Initiation Protocol (SIP) is more and more popular. However, there are many security problems in the Session Initiation Protocol. SIP authentication mechanism is based on HTTP Digest authentication, which this scheme Is insecure; such as off-line password guessing attacks and impersonate other parties and etc. So, researches proposed different schemes to secure the SIP authentication. In the year 2012, Tang et al. proposed a SIP authentication protocol using elliptic curve cryptography (ECC), but their scheme is insecure against off-line password guessing and Registration attacks. In order to overcome such security problems proposed an ECCbased authentication scheme for SIP and analysis of security of the ECC-based protocol.

Keywords - session initiation protocol, Elliptic curve cryptography, Authentication, vulnerability, insecure
\end{abstract}

\section{INTRODUCTION}

Session Initiation Protocol (SIP) proposed by Internet Engineering Task Force (IETF) for the IP-based telephony $[14,15]$.SIP controls communications on the Internet for establishing, maintaining and terminating sessions.SIP is an application -layer control protocol that is a text based protocol and can be used for controlling multimedia communication sessions such as voice and video calls over Internet protocols.[17]. SIP is the one important protocol because of the widespread application of the voice over IP (VoIP)in the Internet so the security of SIP is becoming too important[22].

SIP is a request-response protocol when a user wants to access a SIP service, at the first she/he has to authenticate with SIP server but the original authentication scheme for SIP doesn't provide enough security because it's based on HTTP Digest authentication noted in RFC2617 [8].

Different SIP authentication schemes have been proposed, especially based on Elliptic curve cryptography (ECC) to provide security for SIP. In 2005, Yang et al. found that the original SIP authentication scheme was vulnerable to off-line password guessing attack and server-spoofing attack [19] so they proposed scheme was based on Diffie-Hellman key exchange algorithm [5], which depended on the difficulty of Discrete Logarithm Problem (DLP) [10] but Yang et al.'s scheme was vulnerable to stolen-verifier attack, off-line password guessing attack, and Denning-Sacco attack [4] and Their scheme was high computation cost $[6,9,16]$. In the same year, Based on Yang et al.'s scheme, Durlanik et al. [6] introduced another SIP authentication by using Elliptic Curve Diffie-Hellman (ECDH) key exchange algorithm but this scheme in comparison with Yang et al.'s scheme reduced the execution time and memory requirements. However, their scheme still vulnerability from offline dictionary attack and Denning-Sacco attack [12]. In 2008, Tsai [16] proposed SIP authentication scheme based on random nonce. In this scheme all the communication messages were computed with one-way hash function and exclusive-or operation so computation cost reduce highly. But this scheme vulnerable to off-line password guessing, Denning-Sacco and stolen-verifier attacks; furthermore, it did not provide any key agreement, known-key secrecy and perfect forward secrecy (PFS) [2, 3, 11, 20].In 2009, Wu et al. [23] suggested an SIP authentication scheme based on elliptic curve cryptography (ECC). This scheme achieves authentication and a shared secrecy at the same time. Wu et al.'s scheme provides provable security in the Canetti-Krawczyk (CK) security model [13] and it's suitable for applications that require low memory and rapid transactions. But Wu et al.'s SIP authentication schemes are still vulnerable to off-line password guessing attacks, Denning-Sacco attacks, and stolen-verifier attacks $[10,11]$. In 2009, Yoon et al. proposed another authentication for SIP using ECC in [20]. Unfortunately, the scheme was vulnerable to password guessing attack and stolen-verifier attack. The attack method could be referred to [18]. In 2010, Yoon et al. proposed the third and fourth ECC-based authentication scheme for SIP [21, 24]. But these schemes were vulnerable to offline password guessing and stolen-verifier attacks [18]. In 2011, Arshad et al. proposed SIP authentication scheme based on ECC [2]. ButArshad et al.'s authentication scheme was vulnerable to off-line password guessing attack [1]. In 2012, Tang et al. proposed a secure and efficient authentication scheme based on Elliptic Curve Discrete Logarithm Problem (ECDLP) for SIP. This paper demonstrates the Tang et al.'s authentication scheme vulnerable to off-line password guessing attack and registration attack in this paper and then propose a secure SIP authentication scheme based on ECC in order to solve those security problems. The proposed SIP authentication scheme can provide high security and executes faster than previously proposed schemes.

The remainder of this paper is outlined as follows. Section 2 reviews the original SIP authentication procedure. Section 3 introduces of Tang et al.'s scheme and discusses attack on it and in Section 4 proposed ECC-based mutual authentication scheme for SIP is presented. In section 5 discuss the security and efficiency of the proposed scheme, In Section 6, evaluate the performance of the proposed scheme. And Section 7 is the conclusion.

\section{SIP AUTHENTICATION PROCEDURE}

The common authentication scheme for SIP is Digest Access Authentication (DAA) [8]. DAA security is based on the challenge-response pattern, and this mechanism relies on a shared secret between client and server [19] so Client pre-shares a password with the server before the authentication procedure starts. Fig. 1 shows procedure of the DAA mechanism in SIP. (1) Client $\rightarrow$ Server: REQUEST

The client sends a REQUEST to the server.

(2) Server $\rightarrow$ Client: CHALLENGE (nonce, realm)

The server generates a CHALLENGE that includes a nonce and the client's realm. Then the server sends a CHALLENGE to the 
client.

(3)Client $\rightarrow$ Server: RESPONSE (nonce, realm, username, response)

The client computes a response $=\mathrm{F}$ (nonce, realm, username, response). Note that $\mathrm{F}($.) is a one-way hash function. It is used to generate a digest authentication message, and then the client sends the RESPONSE to the server.

(4) According to the username, the server extracts the client's password. Then the SIP server must perform the same digest operation, and compare the result. If the results are identical, the client is authenticated and a $200 \mathrm{OK}$ message is sent.

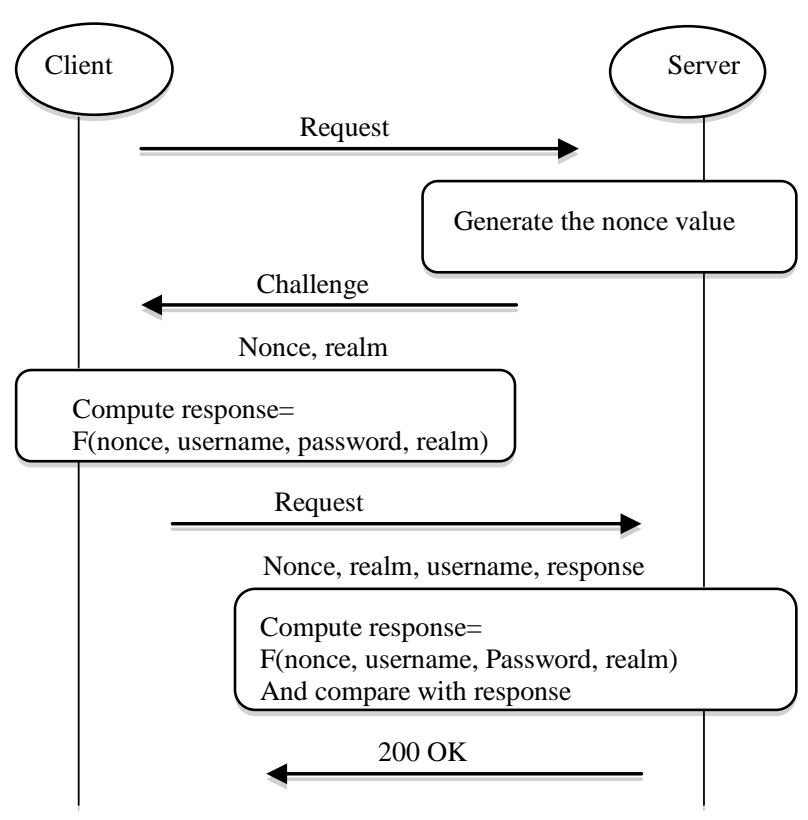

Figure 1. The SIP Digest Access Authentication method during a SIP REGISTER transaction [8]

\section{SIP AUTHENTICATION SCHEME BY TANG ET AL.}

This section reviews Tang et al.'s SIP authentication scheme [1]. Then shows that the scheme is vulnerable to off-line password guessing attacks and registration attacks. Tang et al.'s scheme consists of four phases: system setup phase, registration phase, login and authentication phase, and password change phase.

\subsection{Tang et al.'s scheme}

Tang et al. propose an enhanced ECC-based SIP authentication scheme in order to strength Arshad et al.'s scheme. Notations used in this paper are defined in Table 1.
Table 1.Notations and their explanations [1]

\begin{tabular}{|ll|}
\hline $\mathrm{Ui}, \mathrm{U}:$ & the ith user or user \\
IDi, username: & the identity of user Ui \\
PWi: & Password of user \\
S: & the remote server \\
Ks: & the secret key of the server \\
SK: & a session key \\
$\mathrm{Q}=$ Ks.P: & the public key of the server \\
$\mathrm{h}():$. & a strong cryptographic one-way hash function \\
$\mathrm{H}():$. & a function which makes a point map to another \\
point on elliptic curve \\
ll: & the string concatenation operation \\
$\oplus:$ & the exclusive-or operation \\
$\Rightarrow:$ & a secure channel \\
$\rightarrow:$ & a common channel \\
A $:=B:$ & compares whether A equals B \\
D: & a uniformly distributed dictionary of size lDl \\
\hline
\end{tabular}

\subsubsection{System setup phase}

First the members and the server agree on the EC parameters Then server selects a secret key KS, computes $\mathrm{Q}=\mathrm{KS}$. P and keeps secret KS and publishes p, a, b, P, n, h, Q[1].

\subsubsection{Registration phase}

In this phase:

(1) The user chooses his or her identity IDiand password PWi, then through a pre-established secure channel, such as Virtual Private Network (VPN) or Secure Sockets Layer (SSL) sends them to the server.

(2) The server computes $\mathrm{Vi}=\mathrm{h}($ IDillKS) $\oplus \mathrm{PWiand}$ stores (IDi, Vi) in its database.

\subsubsection{Login and authentication phase}

Fig. 2 illustrates Tang et al.'s SIP authentication scheme. When a legal SIP client $U$ wants to login the SIP server S, the authentication scheme between $\mathrm{U}$ and $\mathrm{S}$ proceeds as follows.

(1) Ui $\rightarrow$ S : REQUEST (IDi, R1)

Ui selects a random nonce $r 1 \in Z_{n}^{*}$ then computesR $=r 1$. P, R1= $\mathrm{R}+\mathrm{H}(\mathrm{IDi}, \mathrm{PWi})$. And then sends message REQUEST (IDi , R1) To the server $\mathrm{S}$.

(2) S $\rightarrow$ Ui : CHALLENGE (S, R2, V1)

First $\mathrm{S}$ checks IDi is exist in database. If yes,Scomputes $\mathrm{PWi}=$ Vi $\oplus$ h(IDill KS $), R^{\prime}=\mathrm{R} 1-\mathrm{h}(\mathrm{IDi}, \mathrm{PWi})=\mathrm{r} 1$. P. And then $\mathrm{S}$ selects arandom nonce $\mathrm{r} 2 \in \mathrm{Z}_{\mathrm{n}}{ }^{*}$, computes $\mathrm{R} 2=\mathrm{r} 2 . \mathrm{P}, \mathrm{SKS}=\mathrm{r} 2$. $\mathrm{R}^{\prime}=\mathrm{r} 1$.r2. $\mathrm{P}, \mathrm{V} 1=\mathrm{h}\left(\mathrm{S}\right.$ llIDillR $\left.11 \mathrm{R}_{2} 1 \mathrm{~S} \mathrm{SK}_{\mathrm{s}}\right)$, and sends CHALLENGE (S, R2, V1) to Ui. At the end, S computesthe common session key SK = h(IDill S 11 R' $11 \mathrm{R}_{2} 11 \mathrm{SK}_{\mathrm{s}} 11 \mathrm{PW}$ )

(3)Ui $\rightarrow$ S : RESPONSE (IDi, S, V2)

Ui computesSKU $=r 1 . R 2=r 1 . r 2 . P$ and checks $V 1$ is equal to $\mathrm{h}(\mathrm{S}$ llIDillRll R2 $11 \mathrm{SKU})$. If they are equal, Ui authenticates the server andcomputes $\mathrm{V} 2=\mathrm{h}$ (IDillS llSKU llPWi). Then Uisends message RESPONSE (IDi, S, V2) Tothe server. ThenUicomputes the session key SK = h(IDill S llR llR2 11 SKU llPWi).

(4)Upon receiving the response message, $\mathrm{S}$ checks $\mathrm{V} 2$ is equal to h(IDillS llSKS llPWi). If the result is equal $\mathrm{S}$ authenticates the identity of Ui andThe common session key is:

$\mathrm{SK}=\mathrm{h}(\mathrm{ID}$ illS 1lr1 .P 1lr2 .P 1lr1 .r2.P 1lPWi) 


$$
\begin{aligned}
& \mathrm{r}_{1} \in \mathrm{Z}_{\mathrm{n}}{ }^{*}, \mathrm{R}=\mathrm{r}_{1} . \mathrm{P} \\
& \mathrm{R}_{1}=\mathrm{R}+\mathrm{H}\left(\mathrm{ID}_{\mathrm{i}} 11 \mathrm{PW} \mathrm{W}_{\mathrm{i}}\right) \\
& \underset{\text { Request }\left(\mathrm{ID}_{\mathrm{i}}, \mathrm{R}_{1}\right)}{\longrightarrow} \\
& \text { Check whether } \mathrm{ID}_{\mathrm{i}} \text { is valid } \\
& \mathrm{PW}_{\mathrm{i}}=\mathrm{V}_{\mathrm{i}} \oplus \mathrm{h}\left(\mathrm{ID}_{\mathrm{i}} 1 \mathrm{llks}\right) \\
& \mathrm{R}^{\prime}=\mathrm{R}_{1}-\mathrm{H}\left(\mathrm{ID}_{\mathrm{i}} 1 \mathrm{lPW} \mathrm{W}_{\mathrm{i}}\right) \\
& \mathrm{r}_{2} \in \mathrm{Z}_{\mathrm{n}}^{*}, \mathrm{R}_{2}=\mathrm{r}_{2} \cdot \mathrm{P} \\
& \mathrm{SK}_{\mathrm{s}}=\mathrm{r}_{2} \cdot \mathrm{R}^{\prime} \\
& \mathrm{V}_{1}=\mathrm{h}\left(\mathrm{S} 11 \mathrm{IID} \mathrm{D}_{\mathrm{i}} 11 \mathrm{R} ' 11 \mathrm{R}_{2} 11 \mathrm{SK}_{\mathrm{s}}\right) \\
& \text { Challenge }\left(\mathrm{S}, \mathrm{R}_{2}, \mathrm{~V}_{1}\right)
\end{aligned}
$$

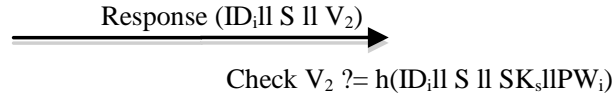

Figure 3. Login and authentication phase [1]

\subsubsection{Password change phase}

In this phase user can change his or herpassword. Figure 3shows the password change phase.

- Server authenticatesUiwith his or her old password PWi.

- After receiving the successful authentication, Uiinputs the new password $\mathrm{PW}_{\mathrm{i}}^{*}$.

- Finally, the server computes $\mathrm{V}_{\mathrm{i}}^{*}$ and replaces $\mathrm{Vi}$ with $\mathrm{V}_{\mathrm{i}}^{*}$.

$\mathrm{U}_{\mathrm{i}}$

Input $\mathrm{ID}_{\mathrm{i}}, \mathrm{PW}_{\mathrm{i}}$

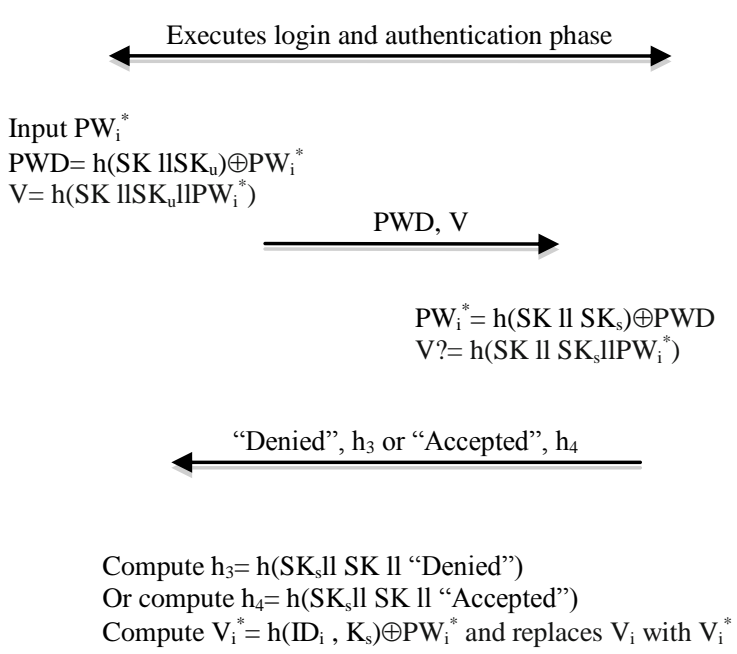

Figure 2. Password change phase [1]

\subsection{Attacks on Tang et al.'s scheme}

In this section shows that Tang et al.'s scheme is vulnerable to off-line password guessing attack and registration attack.

\subsubsection{Off-line password guessing attack}

Off-line password guessing attack works when an attacker tries to find a long-term private key (pw) of Ui.In Tang et al.'s SIP authentication scheme, the off-line password guessing attack is possible.

1. An attacker records Tang et al.'s SIP authentication scheme between the SIP client and the server (use $R_{1}$, $\mathrm{R}_{2}$ and $\mathrm{V}_{2}$ values).

2. By performing the following can run off-line password guessing attack.

a) An attacker selects a candidate password PWi* from the password dictionary D.

b) Attacker Calculate $\mathrm{H}\left(\mathrm{ID}_{\mathrm{i}} 1 \mathrm{lPW} \mathrm{i}_{\mathrm{i}}^{*}\right)$ then $\mathrm{R}_{1}$ $=\mathrm{R}_{1}-\mathrm{H}\left(\mathrm{ID}_{\mathrm{i}} 11 \mathrm{PW} \mathrm{W}_{\mathrm{i}}^{*}\right)=\mathrm{R}+\mathrm{H}\left(\mathrm{ID}_{\mathrm{i}} 11 \mathrm{PW} \mathrm{W}_{\mathrm{i}}\right)-\mathrm{H}$ $\left(\mathrm{ID}_{\mathrm{i}} 11 \mathrm{PW} \mathrm{i}_{\mathrm{i}}^{*}\right)$ output of this step is $\mathrm{R}$.

c) Attacker computes $\mathrm{Sku}^{*}=\mathrm{r}_{1} \cdot \mathrm{R}_{2}$ and $\mathrm{V}_{2}{ }^{*}=\mathrm{h}$ $\left(\mathrm{ID}_{\mathrm{i}} 11 \mathrm{~S} 11 \mathrm{sk}_{\mathrm{u}}{ }^{*} 1 \mathrm{lpw}_{\mathrm{i}}{ }^{*}\right)$

d) Finally compares $\mathrm{V}_{2} *$ with $\mathrm{V}_{2}$. If they are equal, attacker guesses the correct password of $\mathrm{U}$, If not, the attacker repeats the above process until $\mathrm{V}_{2} *=\mathrm{V}_{2}$.

\subsubsection{Registration attack}

During the SIP REGISTER handshake between the UA and the SIP server, authentication process is performed. In this section indicate the shortcomings of Tang et al.'s scheme authentication and recommend whichvalue it requires to be protected.

During the authentication,UA's IP-address is sent in clear and is not protected by Tang et al.'s scheme authentication.Therefore an attacker can modify the hostname or IP-address in real-timethen UA's phone number with the attackers IP address is registered, see figure 4. So After a successful authentication, the attacker's hostname or IP-address is registered in the SIP server. Therefore all requeststo the valid UA will be diverted to a hostname or IP-address set by an attacker. Finally,avalid useris unreachable and server will not detect that anything is wrong, sowhenserver receives a call to validUA, the call will be forwarded to the attackers registered IP address.

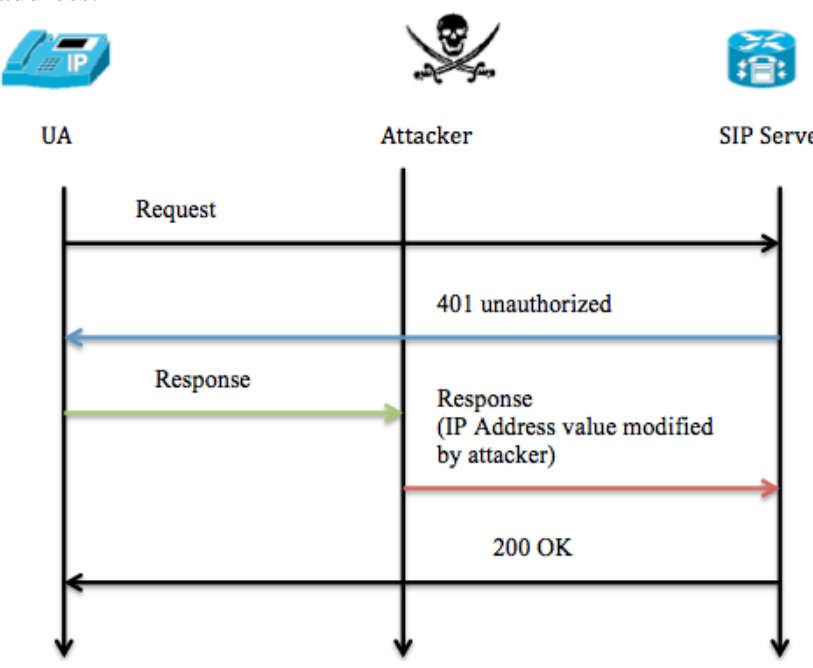

Figure 4.The attacker can modify the IP address value, and all UA's calls redirected to him

\section{Proposed SIP authentication scheme}

This section proposes a new secure SIP authenticationscheme based on elliptic curve cryptography (ECC) to overcome the security problems. Thisscheme is in order to Tang et al.'s scheme. The proposed scheme exploits speed, and security jointly. The proposed scheme consistsof four phases: system setup phase, registration phase, login and authentication phase, and password change phase. 


\subsection{System setup phase}

$\mathrm{U}$ and $\mathrm{S}$ agree on the EC parameters. The server selects a secret key KS, computes $\mathrm{Q}=\mathrm{KS} . \mathrm{P}$, keeps secret $\mathrm{KS}$ and publishes $\mathrm{p}, \mathrm{a}, \mathrm{b}, \mathrm{P}, \mathrm{n}, \mathrm{h}, \mathrm{Q}$

\subsection{Registration phase}

\section{In this phase:}

(1) The user chooses his or her identity IDiand password PWi, then through a pre-established secure channel, such as Virtual Private Network (VPN) or Secure Sockets Layer (SSL) sends them to the server.

(2) The server computes $\mathrm{Vi}=\mathrm{h}($ IDillKS $) \oplus \mathrm{PWiand}$ stores (IDi, Vi) in its database.

This phase is the same Tang et al.'s scheme.

\subsection{Login and authentication phase}

$\mathrm{U}_{\mathrm{i}}$

$\mathrm{r}_{1} \in \mathrm{Z}_{\mathrm{n}}^{*}, \mathrm{R}=\mathrm{r}_{1} \cdot \mathrm{P}$

$\mathrm{R}_{1}=\mathrm{R}+\mathrm{H}\left(\mathrm{IP}_{\mathrm{i}} 11 \mathrm{PW} \mathrm{W}_{\mathrm{i}}\right)$

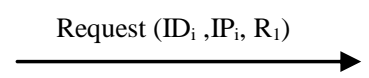

Check whether $\mathrm{ID}_{\mathrm{i}}$ is valid

$\mathrm{PW}_{\mathrm{i}}=\mathrm{V}_{\mathrm{i}} \oplus \mathrm{h}\left(\mathrm{ID}_{\mathrm{i}} 1 \mathrm{lks}\right)$

$\mathrm{R}^{\prime}=\mathrm{R}_{1}-\mathrm{H}\left(\mathrm{IP}_{\mathrm{i}} 11 \mathrm{PW} \mathrm{W}_{\mathrm{i}}\right)$

$\mathrm{r}_{2} \in \mathrm{Z}_{\mathrm{n}}{ }^{*}, \mathrm{R}_{2}=\mathrm{r}_{2} \cdot \mathrm{P}$

$\mathrm{R}_{2}{ }^{*}=\left(\mathrm{r}_{2} \cdot \mathrm{P}\right) \oplus \mathrm{r}_{1}$

$\mathrm{SK}_{\mathrm{s}}=\mathrm{r}_{2} \cdot \mathrm{R}$

$\mathrm{V}_{1}=\mathrm{h}\left(\mathrm{S} 11 \mathrm{ID} \mathrm{D}_{\mathrm{i}} 11 \mathrm{R}\right.$ 'll $\left.\mathrm{R}_{2} 11 \mathrm{SK}_{\mathrm{s}}\right)$

Challenge $\left(\mathrm{S}, \mathrm{R}_{2}{ }^{*}, \mathrm{~V}_{1}\right)$

$\mathrm{R}_{2}{ }^{\prime}=\mathrm{R}_{2}{ }^{*} \oplus \mathrm{r}_{1}=\mathrm{r}_{2} \cdot \mathrm{P}$

$\mathrm{SK}_{\mathrm{u}}=\mathrm{r}_{1} \cdot \mathrm{R}_{2}$

Check $V_{1} ?=\mathrm{h}\left(\mathrm{S} 11 \mathrm{ID} \mathrm{D}_{\mathrm{i}} 11 \mathrm{R} 11 \mathrm{R}_{2}{ }^{\prime} 11 \mathrm{SK}_{\mathrm{u}}\right)$

$\mathrm{V}_{2}=\mathrm{h}\left(\mathrm{ID}_{\mathrm{i}} 11 \mathrm{~S} 11 \mathrm{SK} \mathrm{u}_{\mathrm{u}}\right)$

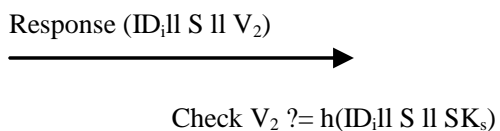

Shared session key $\mathrm{Sk}=\mathrm{r}_{1} \cdot \mathrm{r}_{2} \cdot \mathrm{P}$

Figure 5. Proposed Login and authentication phase

When a legal SIP client U wants to login the SIP server S, the authentication scheme between $\mathrm{U}$ and $\mathrm{S}$ proceeds. Indicated that Tang et al.'s scheme is vulnerable to off-line password guessing attack and registration attack for overcome these security problems, Fig. 5 illustrates the new proposed SIP authentication scheme.

(1) $\mathrm{Ui}_{\mathrm{i}} \rightarrow \mathrm{S}: \mathrm{REQUEST}\left(\mathrm{IDi}, \mathrm{IP}_{\mathrm{i}}, \mathrm{R} 1\right)$

Ui selects a random nonce $r 1 \in Z_{n}^{*}$ then computes $R=r 1$. P, R1 $=\mathrm{R}+\mathrm{H}(\mathrm{IP} i l l P W i)$. And then sends message REQUEST $\left(\right.$ IDi, $\mathrm{IP}_{\mathrm{i}}$ , R1) to the server $\mathrm{S}$.

(2) $\mathrm{S} \rightarrow \mathrm{Ui}$ : CHALLENGE (S, R2*, $\mathrm{V}_{1}$ )

First $\mathrm{S}$ checks IDi is exist in database. If yes, $\mathrm{S}$ computes $\mathrm{PWi}=$ $\mathrm{Vi} \oplus \mathrm{h}(\mathrm{IDill} \mathrm{KS}), \mathrm{R}^{\prime}=\mathrm{R} 1-\mathrm{H}(\mathrm{IP}$ illPWi $)=\mathrm{r} 1$. P. And then $\mathrm{S}$ selects a random nonce $r 2 \in Z_{n}{ }^{*}$, computes $R 2=r 2 . P, R_{2}{ }^{*}=$ $\left(r_{2} \cdot P\right) \oplus r_{1}, S K S=r 2 . R=r 1 . r 2 . P, V_{1}=h\left(S l_{1}^{\prime} D_{i} l l R^{\prime} 11 R_{2} l l\right.$ $\mathrm{SKs})$ and sends CHALLENGE $\left(\mathrm{S}, \mathrm{R}_{2}{ }^{*}, \mathrm{~V}_{1}\right)$ to $\mathrm{Ui}$.
(3) Ui $\rightarrow$ S : RESPONSE (IDill S ll V2)

Ui computes $R_{2}{ }^{\prime}=R_{2}{ }^{*} \oplus r_{1}=\left(r_{2} \cdot P\right) \oplus r_{1} \oplus r_{1}=r_{2} \cdot P, S_{u}=r_{1} \cdot R_{2}$ and Checks $V_{1}$ is equal to $h\left(S 11 I D_{i} 11 R 11 R_{2} 11 S_{u}\right.$ ) If the result is equal Ui authenticates the identity of $\mathrm{S}$. And then computes $\mathrm{V}_{2}=\mathrm{h}\left(\mathrm{ID}_{\mathrm{i}} 1 \mathrm{ll} \mathrm{S} \mathrm{llSK}_{\mathrm{u}}\right.$ ) and sends RESPONSE (IDill S $\left.11 \mathrm{~V} 2\right)$ to the server.

(4) Upon receiving the response message, $S$ checks V2 is equal toh( $\left(\mathrm{ID}_{\mathrm{i}} 1 \mathrm{~S}\right.$ ll $\left.\mathrm{SK}_{\mathrm{s}}\right)$. If the result is equal $\mathrm{S}$ authenticates the identity of Ui.

Shared session key $\mathrm{Sk}=\mathrm{r}_{1} \cdot \mathrm{r}_{2} \cdot \mathrm{P}$

\subsection{Password change phase}

In this phase user can change his or her password. Figure 3shows the password change phase.

- Uineeds to authenticate with his or her old password PWi. After successful authentication, Uiinputs the new password $\mathrm{PW}_{\mathrm{i}}^{*}$.

- $\quad \mathrm{U}_{\mathrm{i}} \rightarrow \mathrm{S}: \mathrm{PWD} ; \mathrm{V}$

$\mathrm{U}_{\mathrm{i}}$ computes $\mathrm{PWD}=\mathrm{h}\left(\mathrm{SK} 11 \mathrm{SK}_{\mathrm{u}}\right) \oplus \mathrm{PW}_{\mathrm{i}}^{*}$ and $\mathrm{V}=\mathrm{h}$

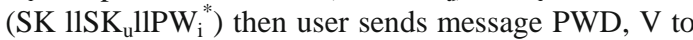
the server.

- $\quad$ Then server computes $\mathrm{PW}_{\mathrm{i}}{ }^{*}=\mathrm{h}\left(\mathrm{SK} l 1 \mathrm{SK}_{\mathrm{s}}\right) \oplus \mathrm{PWD}$ and checks if $\mathrm{V}$ is equal to $\mathrm{h}\left(\mathrm{SK} 11 \mathrm{SK}_{\mathrm{s}} 1 \mathrm{lPW} \mathrm{PW}_{\mathrm{i}}^{*}\right)$ then the server computes $\mathrm{h}_{4}=\mathrm{h}\left(\mathrm{SK}_{\mathrm{s}} \mathrm{ll} \mathrm{SK} 11\right.$ "Accepted") and sends ("Accepted", $\left.\mathrm{h}_{4}\right)$ else computes $\mathrm{h}_{3}=\mathrm{h}\left(\mathrm{SK}_{\mathrm{s}} \mathrm{ll}\right.$ SK 11 "Denied") and sends ("Denied", $\mathrm{h}_{4}$ ) to the user. Finally, the server computes $\mathrm{V}_{\mathrm{i}}^{*}=\mathrm{h}\left(\mathrm{ID}_{\mathrm{i}} 11 \mathrm{~K}_{\mathrm{s}}\right) \oplus \mathrm{PW}_{\mathrm{i}}{ }^{*}$ and replaces $V_{i}$ with $V_{i}^{*}$. This phase is the same Tang et al.'s scheme.

\section{SECURITY ANALYSIS}

This section analyzes a security of the proposed SIP authentication scheme.

\subsection{Define security terms}

- Password (pw): is a low entropy value that can be guessed in polynomial time.

- $\quad \mathrm{K}_{\mathrm{s}}$ (secret key $\mathrm{S}$ ): is a high entropy value that cannot be guessed in polynomial time.

- The Elliptic Curve Discrete Logarithm Problem (ECDLP): Given a public key point $\mathrm{Q}=\alpha \mathrm{P}$, it is hard to compute the secret key $\alpha$.

- The Elliptic Curve Diffie-Hellman Problem (ECDHP): Given point elements $\alpha \mathrm{P}$ and $\beta \mathrm{P}$, it is hard to find $\alpha \beta P$.

- A secure one-way hash function $y=F(x)$ : when given $\mathrm{x}$ it is easy to compute $\mathrm{y}$ and when given $\mathrm{y}$ it is hard to compute $\mathrm{x}$.

\subsection{Security properties}

In this section analyzes the security properties in the proposed SIP authentication scheme.

1. Password guessing attacks

The proposed scheme can resist Password guessing attacks. Off-line password guessing attack:If Eve try to find a PW by repeatedly guessing possible passwords and verifying the correctness of the guesses based on gain information in an offline manner cannot success because In this scheme, all knowledge Eve can gain are $\mathrm{R}_{1}=\mathrm{R}+\mathrm{H}\left(\mathrm{IP}_{\mathrm{i}} 11 \mathrm{PW} \mathrm{W}_{\mathrm{i}}\right), \mathrm{R}_{2}{ }^{*}=\left(\mathrm{r}_{2} \cdot \mathrm{P}\right)$ $\oplus \mathrm{r}_{1}, \mathrm{~V}_{1}$ and $\mathrm{V}_{2}$. Therefore if Eve guessing possible password and computes $\mathrm{H}$ (IP $\mathrm{il} \mathrm{PW})$, it doesn't have any information to compare the guess password is correct or not also in this scheme Eve cannot compute SK because it's difficult Eve breaks the ECDLP and ECDHP, in addition bothr $r_{1}$ and $r_{2}$ values are protected too (see $\mathrm{R}_{1}$ and $\mathrm{R}_{2}{ }^{*}$ ).

On-line password guessing attack: cannot succeed, since $\mathrm{S}$ can choose appropriate the secret key $\mathrm{K}_{\mathrm{s}}$ that is a high entropy number and cannot be guessed by anyone soEvecannot make a successful guess of the right password from $V_{i}$ without the secret 
key Ks of server. Therefore, the proposed scheme can resist against the password guessing attacks.

2. Replay attacks

The proposed scheme can resist the replay attacks. Ifan attacker replays REQUEST to impersonate U in Step (1),in Step (3), Eve cannot compute a correct session key sk and deliver it to $\mathrm{S}$, so the proposed scheme can resist against the replay attacks.

3. Man-in-the-middle attacks

The proposed scheme can resist against the man-in-the-middle attacks because this scheme based on mutual authentication and shared secret PWbetween U and S so PWi of Ui and the secret key Ks of S are used to prevent the man-in-middle attack.

4. Stolen-verifier attacks

The proposed scheme can resist against the stolen-verifier attacks because servers are always the targets of attacks but in this scheme the server computes $\mathrm{Vi}=\mathrm{h}$ (IDillKS $) \oplus \mathrm{PWiand}$ stores (IDi, Vi) in its database so if attacker Eve steals verifier from the database of the server,Eve cannot make a successful guess of the right password from Vi .because Eve doesn't have thesecret key $\mathrm{K}_{\mathrm{s}}$ of server and also $\mathrm{K}_{\mathrm{s}}$ is a high entropy number.

5. Impersonation attacks

The proposed scheme can resist against the impersonation attacks. In this scheme attacker Eve cannot masquerade as server because doesn't have $\mathrm{K}_{\mathrm{s}}$ and on the other hand Eve cannot masquerade as U because doesn't have knowledge about PW.

6. Oracle attacks

The proposed scheme can resist against the oracle attacks because in this scheme doesn't use decryption oracle.

7. Denning-Sacco attacks

The proposed scheme can resist against Denning-Sacco attacks.Assume that attacker Eve may find fresh session key SK for some reasons. But Eve cannot detectPWi and server's secret key Ks because $S K=r_{1} \cdot r_{2} \cdot P$ and it's ECDHP so Eve cannot compute.

8. Registration attacks

The proposed scheme can resist against registration attacks. In this scheme by hashing IP address of $U_{i}$ in phase (1), protected this value. Therefore, the proposed scheme can resist against the registration attacks.

9. Known-key security

The proposed scheme provides Known-key security, means that during authentication between $\mathrm{U}_{\mathrm{i}}$ and $\mathrm{S}$, they should produce unique secret session keyby random values $r_{1}$ and $r_{2}$.

10. Session key security

The proposed scheme provides session key security because of ECDLP, ECDHP and secure one-way hash function thereforejust $\mathrm{U}_{\mathrm{i}}$ and $\mathrm{S}$ can compute the session key at the end of the key exchange.

\section{Perfect forward secrecy}

The proposed scheme provides PFS means that, if long-term private keys such as user's passwordPW $\mathrm{W}_{\mathrm{i}}$ and secret key $\mathrm{K}_{\mathrm{s}}$ of serverare compromised, there isn't any effect on the secrecy of previous session keys. Because of ECDHP and attacker Eve cannot compute $\mathrm{Sk}=\alpha \beta \mathrm{P}$ from $\mathrm{R}_{1}$ and $\mathrm{R}_{2}{ }^{*}$.

12. Mutual authentication

The proposed scheme provides mutual authentication means that during authentication mechanism both the user and the server are authenticated each other. Server can authenticate the user by checking whether $\mathrm{V}_{2}$ is correct and $\mathrm{U}_{\mathrm{i}}$ can authenticate the identity of the server if $\mathrm{V}_{1}$ is correct.

The security properties of the previously reported schemes $[1,2,6,16,19,21]$ and the proposed scheme are summarized in Table 2 .

\section{PERFORMANCE COMPARISONS}

The computation costs of proposed scheme and the previously schemes $[1,2,6,16,19,21]$ are shown in Table 3 .The proposed SIP authentication scheme requires four PM (elliptic curve scale multiplication), two HP (hash-to-point function), two $\mathrm{PA}$ (point addition) and five $\mathrm{H}$ (hash function operations) during the protocol execution. The proposed scheme is efficient authentication schemes for Session Initiation Protocol.

The proposed SIP authentication scheme requires elliptic curve scale multiplication computations and hash-to-point operations to resist the password guessing attack and provide known-key secrecy and PFS.

\section{CONCLUSIONS}

This paper indicates the vulnerabilities of Tang et al.'s authentication schemes for session initiation protocol (SIP) to off-line password guessing attacks and registration attacks. In order to resolve the shortcomings in their scheme, proposed a new secure and efficient SIP authenticationscheme. The proposed scheme based on ECC, resists the mentioned attacks. Through the performance comparisons, we have shown that the proposed scheme is more efficient and needs to compute four elliptic curve scale multiplications and two hash-to-point function operations during a protocol run so by using this method can decrease computational time for authentication phase in SIP. At the end,the proposed scheme maintains high efficiency and security compared with previous ECC-based authentication schemes.

Table 2.Comparisons of the security properties of different schemes

\begin{tabular}{|c|c|c|c|c|c|c|c|}
\hline & Durlanik[6] & Yang [19] & Tsai [16] & Yoon [21] & Arshad [2] & Tang [1] & Ours \\
\hline Impersonation attack & Insecure & Insecure & Insecure & Secure & Insecure & Secure & Secure \\
\hline Password guessing attack & Insecure & Insecure & Insecure & Insecure & Insecure & Insecure & Secure \\
\hline Denning Sacco attack & Insecure & Insecure & Insecure & Secure & Secure & Secure & Secure \\
\hline Stolen-verifier attack & Not applicable & Insecure & Insecure & Insecure & Secure & Secure & Secure \\
\hline Registration attack & Insecure & Insecure & Insecure & Insecure & Insecure & Insecure & Secure \\
\hline Mutual authentication & Provided & Provided & Provided & Provided & Provided & Provided & Provided \\
\hline Session key security & Provided & Not applicable & Provided & Provided & Provided & Provided & Provided \\
\hline Known key secrecy & Provided & Not applicable & Not provided & Provided & Provided & Provided & Provided \\
\hline Perfect forward secrecy & Provided & Not applicable & Not provided & Provided & Provided & Provided & Provided \\
\hline
\end{tabular}


Table 3.Comparison of computation cost

\begin{tabular}{|c|c|c|c|c|c|c|c|}
\hline & Durlanik[6] & Yang [19] & Tsai [16] & Yoon [21] & Arshad [2] & Tang [1] & Ours \\
\hline Exponentiation & $\mathbf{0}$ & $\mathbf{4}$ & $\mathbf{0}$ & $\mathbf{0}$ & $\mathbf{0}$ & $\mathbf{0}$ & $\mathbf{0}$ \\
\hline Scale multiplication & $\mathbf{4}$ & $\mathbf{0}$ & $\mathbf{0}$ & $\mathbf{6}$ & $\mathbf{5}$ & $\mathbf{4}$ & $\mathbf{4}$ \\
\hline Point addition & $\mathbf{0}$ & $\mathbf{0}$ & $\mathbf{0}$ & $\mathbf{3}$ & $\mathbf{0}$ & $\mathbf{2}$ & $\mathbf{2}$ \\
\hline Hash-to-point & $\mathbf{0}$ & $\mathbf{0}$ & $\mathbf{0}$ & $\mathbf{0}$ & $\mathbf{0}$ & $\mathbf{2}$ & $\mathbf{2}$ \\
\hline Hash function & $\mathbf{6}$ & $\mathbf{8}$ & $\mathbf{7}$ & $\mathbf{4}$ & $\mathbf{8}$ & $\mathbf{7}$ & $\mathbf{5}$ \\
\hline Exclusive or & $\mathbf{4}$ & $\mathbf{4}$ & $\mathbf{3}$ & $\mathbf{0}$ & $\mathbf{2}$ & $\mathbf{1}$ & $\mathbf{3}$ \\
\hline Security & ECDLP & DLP & HASH & ECDLP & ECDLP & ECDLP & ECDLP \\
\hline
\end{tabular}

\section{REFERENCES}

[1] Tang H, Liu X (2012) Cryptanalysis of Arshad et al.'s ECC-based mutual authentication scheme for session initiation protocol. Multimed Tools Appl. DOI 10.1007/s11042-012-1001-8

[2] Arshad R, Ikram N (2011) Elliptic curve cryptography based mutual authentication scheme for session initiation protocol. Multimed Tool Appl. doi:10.1007/s11042-0110787-0

[3] Chen TH, Yeh HL, Liu PC, Hsiang HC, Shih WK (2010) A secured authentication protocol for SIP using elliptic curves cryptography. CN, CCIS 119:46-55

[4] Denning D, Sacco G (1981) Timestamps in key distribution systems. Commun ACM 24:533-536

[5] DiffieW, Hellman ME (1976) New directions in cryptography. IEEE Transactions on Information Theory IT-22: 644-654

[6] Durlanik A, Sogukpinar I (2005) SIP Authentication Scheme using ECDH. World EnformatikaSocityTransations on Engineering Computing and Technology 8:350-353

[7] Ryu JT, Roh BH, Ryu KY (2009) Detection of SIP flooding attacks based on the upper bound of the possible number of SIP messages, KSII Transactions on Internet and Information Systems (TIIS) 3 (5) 507-526.

[8] Franks J, Hallam-Baker P, Hostetler J, Lawrence S, Leach P, Luotonen A, Stewart L (1999) HTTP authentication: basic and digest access authentication, IETF RFC2617.

[9] He DB, Chen JH, Zhang R (2011) A more secure authentication scheme for telecare medicine information systems. J Med Syst. doi:10.1007/s10916-011-9658-5

[10] Menezes AJ, Oorschot PC, Vanstone SA (1997) Handbook of Applied Cryptograph,CRC Press.

[11] Lin CL, Hwang T (2003) A password authentication scheme with secure password updating. ComputSecur 22(1):68-72

[12] Yoon EJ, Yoo KY (2009) Cryptanalysis of DS-SIP authentication scheme using ECDH, in 2009 International Conference on New Trends in Information and Service Science 642-647.
[13] Canetti R, Krawczyk H (2001) Analysis of key-exchange protocols and their use for building secure channels, in: Proc. Eurocrpt 2001, Lecture Notes in Computer Science, 2045, pp. 453-474.

[14] Rosenberg J,Schulzrinne H, Camarillo G, Johnstone A, Peterson J, Sparks R (2002) SIP: session initiation protocol. IETF RFC3261

[15] Thomas M (2001) SIP security requirements. IETF Internet Draft (draftthomas-sip-sec-reg-00.txt)

[16] Tsai JL (2009) Efficient nonce-based authentication scheme for session initiation protocol.Int J NetwSecur 8(3):312316

[17] Veltri L, Salsano S, Papalilo D (2002) SIP security issues: the SIP authentication procedure and its processing load. IEEE Netw 16(6):38-44

[18] Xie Q (2011) A new authenticated key agreement for session initiation protocol. Int $\mathrm{J}$ Commun Syst. doi:10.1002/dac. 1286

[19] Yang CC,Wang RC, Liu WT (2005) Secure authentication scheme for session initiation protocol. ComputSecur 24:381-386

[20] Yoon EJ, Yoo KY (2009) A new authentication scheme for session initiation protocol, in 2009 International Conference on Complex, Intelligent and Software Intensive Systems, CISIS '09 549-554

[21] Yoon EJ, Koo KY (2010) Robust mutual authentication with a key agreement scheme for the session initiation protocol. IETE Tech Rev 27(3):203-213

[22] Geneiatakis D, Dagiuklas T, Kambourakis G, Lambrinoudakis C, Gritzalis S, Ehlert S (2006) Survey of security vulnerabilities in session initiation protocol. IEEE CommunSurv Tutorials 8(3):68-81

[23] Wu L, Zhang Y, Wang F (2009) A new provably secure authentication and key agreement protocol for SIP using ECC, Computer Standards and Interfaces 31 (2) 286-291.

[24] Yoon EJ, Yoo KY (2010) A three-factor authenticated key agreement scheme for SIP on elliptic curves, in Proceedings of the 2010 Fourth International Conference on Network and System Security 334-339 